Rev. Adm. Saúde Vol. 17, № 68, Jul. - Set. 2017

http://dx.doi.org/10.23973/ras.68.47

ARTIGO ORIGINAL

\title{
Satisfação dos idosos atendidos pela estratégia de saúde da família em um município do interior de Pernambuco
}

Satisfaction of the elderly seen by the family health strategy in a city in the interior of Pernambuco

\section{Alaine Santos Parente ${ }^{1}$, Fabíola Olinda De Souza Mesquita², Marcos Renato Oliveira $^{3}$}

\begin{abstract}
1. Fisioterapeuta, especialista em gestão em saúde pela Universidade Federal do Vale do São Francisco (UNIVASF) e especialista em saúde da pessoa idosa pela Universidade Federal do Maranhão. Discente da Especialização em Gestão Pública pela UNIVASF.
\end{abstract}

2. Odontóloga, especialista em gestão em saúde pela Universidade Federal do Vale do São Francisco (UNIVASF)

3. Enfermeiro, mestre em enfermagem pela Universidade Federal do Ceará.

\section{RESUMO}

O aumento da população idosa trouxe consigo um grande impacto social, exigindo uma atuação direcionada para este público. É função das políticas de saúde contribuir para que mais pessoas alcancem idades avançadas com o melhor estado de saúde possível, sendo o envelhecimento ativo e saudável, o principal objetivo. A avaliação da satisfação dos usuários constitui-se em uma importante ferramenta para subsidiar o processo de decisão compartilhada, de forma a se repensar as práticas profissionais e reorganizar o processo de trabalho. O presente trabalho apresentou como objetivo avaliar a satisfação dos idosos atendidos pela Estratégia de Saúde da Família no município de Salgueiro, estado de Pernambuco. Foi realizado estudo descritivo de abordagem quantitativa realizado a partir da aplicação de um questionário referente a utilização e acesso ao serviço, aos idosos que estavam em atendimento em cada unidade de saúde. A maioria dos idosos relatou frequentar o serviço mensalmente, avaliou o atendimento como bom, sentiram confiança na equipe, consideraram o tempo de atendimento suficiente e relataram nunca ter dificuldade na marcação das consultas. Quase a totalidade dos participantes afirmou que não participam de grupos na Unidade Básica de Saúde e não tem conhecimento sobre a realização de atividades educativas. A partir da 
pesquisa observou-se que a maioria dos idosos apresenta satisfação quanto ao atendimento, no entanto, destaca-se que é preciso avaliar continuamente as práticas voltadas para esse grupo populacional considerando não apenas as atividades assistenciais, mas também práticas preventivas e de promoção à saúde com uma abordagem multiprofissional e intersetorial.

Palavras-chave: Atenção primária à saúde. Idoso. Satisfação do usuário.

\begin{abstract}
The increase in the elderly population has brought with it a great social impact, requiring a performance directed to this audience. It is the role of health policies contribute to that more people reach advanced ages with the best health status possible, being active and healthy aging, the main objective. The evaluation of user satisfaction is an important tool to subsidize the decision-making process, in order to rethink professional practices and reorganize the work process. The present study had as objective to evaluate the satisfaction of the elderly seen by the Family Health Strategy in the municipality of the Salgueiro- Pernambuco. A descriptive study was carried out with a quantitative approach conducted from the application of a questionnaire concerning the use and access to the service, the elderly who were in attendance at each health unit. Most of the elderly reported attending the service monthly, he evaluated the as good, felt confidence in the team, took the time to care enough and reported never having difficulty in marking of queries. Almost all of the participants said they did not attend groups in Basic Health Unit and has no knowledge about the achievement of educational activities. From the research, it was observed that the majority of the elderly present satisfaction regarding care, however, it is important to point out that it is necessary to continuously evaluate the practices aimed at this population group considering not only the assistance activities but also preventive practices and promotion of the With a multi-professional and intersectoral approach.
\end{abstract}

Keywords: Primary Health Care. Aged. Consumer Satisfaction.

\title{
INTRODUÇÃO
}

O Programa Saúde da Família (PSF) criado e implantado em 1994, constituiu-se em uma importante estratégia para reorganizar as práticas na atenção primária à saúde (APS) e reorientar o sistema de saúde brasileiro, por meio do sistema de referência e contrarreferência. Em suma, tratou-se de um modelo pautado no trabalho em equipe, priorização da família em seu território, acolhimento, vínculo, ações de prevenção e promoção à saúde, tratamento e reabilitação ${ }^{1}$. 
De acordo com o Ministério da Saúde (MS) 2, a atenção básica caracteriza-se por um conjunto de ações de saúde, no âmbito individual e coletivo, que abrange a promoção e a proteção da saúde, a prevenção de agravos, o diagnóstico, o tratamento, a reabilitação, a redução de danos e a manutenção da saúde com o objetivo de desenvolver uma atenção integral que impacte na situação de saúde e autonomia das pessoas e nos determinantes e condicionantes de saúde das coletividades, devendo realizar em seu processo de trabalho ações que incluam diversos públicos, entre eles: crianças, adolescentes, adultos e idosos.

O aumento da população idosa no Brasil e no mundo trouxe consigo um grande impacto social, exigindo uma atuação direcionada para este público. De acordo com - Ministério da Saúde do Brasil ${ }^{3}$, é função das políticas de saúde contribuir para que mais pessoas alcancem idades avançadas com o melhor estado de saúde possível, sendo o envelhecimento ativo e saudável, o principal objetivo.

A Estratégia de Saúde da Família (ESF) possui um importante papel nesse cenário devendo usar em seu processo de trabalho estratégias de prevenção de doenças e promoção da saúde com o objetivo de alcançar um processo de envelhecimento mais saudável e ativo, melhorando assim sua qualidade de vida, devendo incluir diversas atividades para esse grupo populacional, como: alimentação saudável para pessoas idosas; prática corporal/atividade física, trabalho em grupo (grupo de hipertensos, diabéticos, atividade física, entre outros), visita domiciliar, avaliação considerando o seu contexto de vida e social; avaliação global da pessoa idosa abordando diversos aspectos, como os de alimentação e nutrição, comunicação, incontinência, atividades básicas e instrumentais de vida diária, capacidade cognitiva, mobilidade, suporte familiar e social e demais fatores importantes para a manutenção da saúde da pessoa idosa ${ }^{4}$.

Cabe ressaltar ainda que com base no princípio de territorialização, a atenção básica/atenção primária à saúde deve ser responsável pela atenção à saúde de todas as pessoas idosas que estão na sua área de abrangência, inclusive, aquelas que se encontram em instituições públicas ou privadas ${ }^{4}$.

No âmbito do processo de trabalho da atenção básica deve-se considerar ainda a avaliação de suas ações pelos usuários e pelas equipes de trabalhadores. Alguns instrumentos de avaliação como questionários de satisfação com o atendimento vêm sendo aplicados pelas unidades e devem ser cada vez mais utilizados, visto que a aplicação e avaliação das respostas oriundas dos mesmos podem contribuir para manter formas de atuação e/ou formular novas propostas de trabalho além de ajudar os gestores no planejamento e implementação de ações fortalecendo a participação popular nos processos de planejamento e controle social.

Segundo pesquisadores ${ }^{5}$, a satisfação dos usuários tem ocupado um lugar progressivamente mais importante na avaliação da qualidade dos serviços. Esta posição considera que a satisfação está diretamente relacionada à adesão terapêutica e com os resultados dos cuidados em saúde, influenciando comportamentos de saúde e doença, além da crescente valorização do papel do usuário.

A avaliação da satisfação dos usuários dos serviços de saúde constitui-se, portanto, em uma importante ferramenta para subsidiar o processo de decisão compartilhada, 
de forma a colaborar para o processo de repensar as práticas profissionais e reorganizar o processo de trabalho desenvolvido, realocar recursos além de possibilitar readequar ações e redefinir objetivos que estejam coerentes com o projeto de saúde estabelecido ${ }^{6}$. Nessa perspectiva, a avaliação com consequente reorganização do processo de trabalho, proporcionará benefícios aos usuários, promovendo melhor qualidade da atenção à saúde.

A percepção do usuário é considerada de extrema importância ao se dimensionar o reflexo das ações que vem sendo desenvolvidas no setor saúde, servindo como vetor de direcionamento e planejamento do serviço ${ }^{7}$. Considerando então, que a ESF possui em seu processo de trabalho o acolhimento, atendimento e acompanhamento do usuário idoso encontrando-se este usualmente em situação de vulnerabilidade e necessitando deste serviço se faz necessária identificar a satisfação destes indivíduos quanto ao atendimento prestado nessas Unidades de Saúde da Família (USF). Dessa forma, o objetivo da pesquisa foi de avaliar a satisfação dos idosos atendidos pela ESF em um município do interior de Pernambuco.

\section{MATERIAL E MÉTODOS}

Trata-se de estudo descritivo de abordagem quantitativa. O mesmo foi realizado em Unidades de Saúde da Família do município de Salgueiro- PE, onde segundo dados do Instituto Brasileiro de Geografia e Estatística (IBGE) ${ }^{8}$ possui uma população total estimada de 59.409 habitantes.

De acordo com dados do Cadastro Nacional de Estabelecimentos de Saúde/Departamento de Informática do Sistema Único de Saúde (CNES/DATASUS) $^{9}$, o município possui 17 USF cadastradas estando estas localizadas tanto em área urbana (12 USF) quanto rurais (5 USF). Destas 17 unidades foram consideradas apenas aquelas na zona urbana por caracterizar-se em áreas de melhor acesso para realização da pesquisa. Cada USF recebeu a visita da pesquisadora no período de outubro e novembro de 2014 , sendo previamente explicada aos trabalhadores de cada unidade sobre a pesquisa, onde apenas cinco unidades possuindo estas uma equipe de saúde da família em cada unidade, concordaram a realização do estudo.

A população desse estudo correspondeu aos usuários idosos atendidos pelas Unidades de Saúde da Família do referido município onde segundo informações colhidas na Secretaria Municipal de Saúde a partir de dados do Sistema de Informação da Atenção Básica (SIAB) na competência de outubro de 2014, o município possui 5152 pessoas cadastradas acima de 60 anos de idade, sendo que destes indivíduos 2.164 são do sexo masculino e 2.988 do sexo feminino.

A amostra, do tipo de conveniência, foi constituída pelos usuários que durante o período de coleta receberam atendimento na unidade de saúde visitada. Ao final do atendimento os usuários que atenderam aos critérios de inclusão foram solicitados a responder o questionário sendo previamente explicados sobre o objetivo do estudo assinando posteriormente o Termo de Consentimento Livre e Esclarecido (TCLE) declarando responder voluntariamente a pesquisa. 
Participaram da pesquisa, usuários cadastrados nas unidades básicas de saúde; indivíduos com mais de 60 anos de idade que possuam condições em responder ao questionário e que tenham pelo menos uma experiência de atendimento na USF. Foram excluídos deste estudo idosos que apresentavam problemas de fala que impossibilitasse a compreensão dos dados coletados.

Após as visitas as unidades apenas 96 idosos concordaram em responder a pesquisa; O pouco número de idosos conseguido é justificado pelo período de tempo da pesquisa (apenas 2 meses) e a pouca adesão dos usuários em participar da mesma, além do reduzido número de usuários encontrados durante os dias de visita a unidade.

Para realização do estudo foi construído instrumento próprio considerando a satisfação quanto ao acesso para consulta, atendimento, tempo de espera, visita domiciliar, grupos na atenção básica, interação usuário-profissional, sendo estes itens minuciosamente descritos nos parágrafos a seguir.

O questionário utilizado neste estudo permitiu ainda o registro de características sócio-demográficas relativas a sexo; idade e escolaridade; além de avaliar com que frequência o idoso faz uso da unidade de saúde; atendimento recebido pela equipe de saúde da família em variáveis como: ótimo, bom, regular, ruim ou péssimo; se o idoso sentiu confiança durante $o$ atendimento; como classifica o tempo de espera para consulta (demorou um pouco; demorou bastante ou não demorou); se o mesmo encontra dificuldade na marcação das consultas assinalando uma das respostas (sempre; as vezes; raramente ou nunca); se participar de algum grupo na Unidade Básica de Saúde (UBS), se está satisfeito ou não.

Além dessas questões, foi perguntado como o idoso avalia a visita domiciliar e as informações dadas pelo profissional considerando as variáveis: ótima, boa, regular, ruim ou péssima; se a equipe de saúde atende as suas expectativas, atendendo-Ihe com respeito e ética, fornecendo-lhe as informações necessárias e recebendo o devido atendimento e continuidade do mesmo pela equipe; se existe prioridade no atendimento; como considera o tempo de atendimento: rápido, demorado demais ou suficiente, conseguindo receber a atenção necessária. Por fim, foi questionado se 0 usuário já participou de alguma atividade educativa voltada para temas específicos para o idoso (palestra sobre hipertensão, diabetes, prevenção de quedas, alimentação e prática de atividade física), perguntando-se ao mesmo como ele considera essas atividades utilizando as seguintes opções: ótima, boa, regular, ruim, péssima ou não tem conhecimento.

Após a coleta, foi realizada a análise dos dados sendo estes dispostos em tabelas através de estatística descritiva simples utilizando o programa Microsoft Excel 2007.

O estudo respeitou as Diretrizes e Normas Regulamentadoras de Pesquisa em Seres Humanos, Resolução no. 466/12 do Conselho Nacional de Saúde/Ministério da Saúde, sendo aprovado pelo Comitê de Ética e Pesquisa com número de parecer 660.902 do ano de 2014.

\section{RESULTADOS}


A partir da pesquisa foi possível observar características sóciodemográficas destes indivíduos e aspectos relacionados ao atendimento na ESF relativas a frequência de utilização do serviço, avaliação do atendimento, confiança na equipe, tempo de espera para consulta e tempo de atendimento, satisfação quanto a visita domiciliar, práticas grupais, prioridade no atendimento e atividades educativas que serão expostos detalhadamente nos parágrafos a seguir.

Do total de usuários entrevistados percebeu-se que a maioria corresponde ao sexo feminino (65,63\%), com predominância de faixa etária entre 60 a 70 anos de idade $(61,46 \%)$. Em relação à escolaridade, a maioria dos indivíduos entrevistados $(38,54 \%)$ declarou possuir apenas ensino fundamental incompleto (Tabela 1).

Tabela 1. Características sócio-demográficas dos idosos atendidos na Estratégia de Saúde da Família do município de Salgueiro- PE.

\begin{tabular}{|c|c|c|}
\hline & $\mathbf{N}$ & $\%$ \\
\hline \multicolumn{3}{|l|}{ SEXO } \\
\hline Feminino & 63 & 65,63 \\
\hline Masculino & 33 & 34,37 \\
\hline \multicolumn{3}{|l|}{ IDADE } \\
\hline 60 a 70 anos & 59 & 61,46 \\
\hline 70 a 80 anos & 20 & 20,83 \\
\hline Acima de 80 anos & 17 & 17,71 \\
\hline \multicolumn{3}{|l|}{ ESCOLARIDADE } \\
\hline Analfabeto & 29 & 30,21 \\
\hline Fundamental incompleto & 37 & 38,54 \\
\hline Fundamental completo & 10 & 10,42 \\
\hline Ensino médio incompleto & 5 & 5,21 \\
\hline Ensino médio completo & 11 & 11,46 \\
\hline Graduação & 2 & 2,08 \\
\hline Pós-graduação & 2 & 2,08 \\
\hline TOTAL & 96 & 100,00 \\
\hline
\end{tabular}

Fonte: Pesquisa direta, 2014.

Em relação à satisfação do idoso quanto ao atendimento na ESF foram levantados vários questionamentos, dentre estes, destaca-se a frequência de utilização desse serviço, sendo que a maioria $(64,58 \%)$ dos idosos entrevistados relatou frequentar o serviço mensalmente. $\mathrm{O}$ segundo questionamento correspondeu à avaliação sobre 0 atendimento recebido pela equipe, sendo que a maioria relatou como sendo bom $(58,33 \%)$. A maior parte dos usuários destacou ainda possuir confiança na equipe durante 0 atendimento $(88,54 \%)$. No que se refere ao tempo de espera para consulta, verifica-se que a maioria dos usuários relatou que para ser atendido demorou apenas um pouco (37,50\%). Em relação ao tempo de atendimento, a maioria $(69,79 \%)$ declarou ser suficiente para atender as suas queixas (Tabela 2$)$. 
Tabela 2. Satisfação do idoso quanto ao atendimento na Estratégia de Saúde da Família do município de Salgueiro- PE.

\begin{tabular}{lrr}
\hline Questão & N & \% \\
\hline 1- Com que frequência você vem a unidade básica de saúde? & 5 & 5,21 \\
Semanalmente & 62 & 64,58 \\
Mensalmente & 7 & 7,29 \\
Anual & 22 & 22,92 \\
Raramente & & \\
2- Como você avalia o atendimento recebido pela equipe de saúde da & & \\
família? & 13 & 13,54 \\
Ótimo & 56 & 58,33 \\
Bom & 21 & 21,87 \\
Regular & 3 & 3,13 \\
Ruim & 3 & 3,13 \\
Péssimo & & \\
3- Você sentiu confiança na equipe durante o atendimento? & 85 & 88,54 \\
Sim & 11 & 11,46 \\
Não & & \\
4- Como você classifica o tempo de espera para consulta? & 36 & 37,50 \\
Demorou um pouco & 29 & 30,21 \\
Demorou bastante & 31 & 32,29 \\
Não demorou & & \\
5- Como você considera o tempo de atendimento? & 18 & 18,75 \\
Demorado & 11 & 11,46 \\
Rápido & 67 & 69,79 \\
Suficiente & $\mathbf{9 6}$ & $\mathbf{1 0 0 , 0 0}$ \\
TOTAL & & \\
\hline
\end{tabular}

Fonte: Pesquisa direta, 2014.

No que se refere ao questionamento sobre o acesso as consultas na unidade a maioria $(51,04 \%)$ afirmou nunca possuir dificuldade na marcação. Quando questionados se participam de algum grupo na unidade básica de saúde a maioria $(95,83 \%)$ declarou não ter participado sendo que apenas alguns participantes $(4,17 \%)$ relataram estarem satisfeitos com essa atividade. Em relação a visita domiciliar do Agente Comunitário de Saúde (ACS) a maioria dos entrevistados declarou receber a visita mensalmente (93,75\%) avaliando-a como boa $(56,25 \%)$ o que revela que a maioria dos idosos estão satisfeitos com a visita domiciliar recebida (tabela 3).

Os idosos responderam ainda que a equipe de saúde atende as suas expectativas sempre que frequentam a unidade $(82,29 \%)$. Em relação a prioridade no 
atendimento a maior parte dos usuários relataram existir $(61,46 \%)$ e alguns usuários declaram não possuir prioridade (38,54\%), o que sugere que em algumas unidades os idosos apresentam os mesmos critérios para atendimento que os demais usuários de outras faixas etárias. Por fim, foram questionados se já participaram de alguma atividade educativa na unidade de saúde sendo que a maioria declarou não ter conhecimento sobre esse tipo de atividade (57,29\%), caracterizando assim, que nas unidades de saúde estudadas há predomínio de atividades curativas em detrimento de práticas preventivas e de promoção à saúde (tabela 3).

Tabela 3. Satisfação do idoso quanto ao atendimento na Estratégia de Saúde da Família do município de Salgueiro- PE.

\begin{tabular}{lrr}
\hline & N & $\%$ \\
\hline 6- Você encontra dificuldade na marcação da consultas? & N & $\%$ \\
Sempre & 21 & 21,88 \\
Às vezes & 23 & 23,95 \\
Raramente & 3 & 3,13 \\
Nunca & 49 & 51,04
\end{tabular}

7- Participa de algum grupo na unidade básica de saúde?

Sim

Não

7.1- Se sim está satisfeito com as atividades realizadas?

Sim

Não

8- Recebe mensalmente visita domiciliar do ACS?

Sim

Não

8.1 Como você avalia a visita e as informações dadas pelo profissional?

Ótima

Boa

Regular

Ruim

Péssima

9- A equipe de saúde atende as suas expectativas, atendendo-Ihe com respeito e ética, fornecendo-lhe as informações necessárias e recebendo o devido atendimento e continuidade do mesmo pela equipe?

Sempre

Às vezes

Raramente

10- Existe prioridade no atendimento? 
11- Já participou de atividades educativas voltadas para o idoso na unidade? Se sim, como você considera essas atividades?

Ótimo

$\begin{array}{rr}9 & 9,38 \\ 24 & 25 \\ 7 & 7,29 \\ 1 & 1,04 \\ 0 & 0 \\ 55 & 57,29 \\ \mathbf{9 6} & \mathbf{1 0 0 , 0 0}\end{array}$

Bom

Regular

Ruim

Não tenho conhecimento

96100,00

Fonte: Pesquisa direta, 2014.

\section{DISCUSSÃO}

As características sociodemográficas encontradas assemalham-se a de outros estudos realizados sobre a satisfação dos usuários com predominância de mulheres e baixa escolaridade ${ }^{10,11,12,13}$.

A menor participação dos homens pode ser decorrente de um maior vínculo com o trabalho na faixa etária produtiva, buscando assim, alternativas para a assistência, ou por outro lado, pela não priorização a esses usuários ${ }^{13}$.

Mastroeni et al. ${ }^{11}$, em seu estudo também relataram que dos indivíduos entrevistados a maioria (72,9\%) dos que frequentaram a escola revelou não ter completado o ensino fundamental ressaltando que é importante destacar que antigamente havia menor frequência à escola, priorizando-se o trabalho em detrimento da educação. Nessa época, grande parte da população estudava até a quarta série do primário, visto que não havia exigência de maior escolaridade para se conseguir melhores cargos e, consequentemente, melhor renda.

O mesmo autor descreve ainda que o aumento do índice de alfabetização entre os idosos é um fato altamente positivo para esse grupo populacional estando ligado ao maior acesso às informações divulgadas pelos meios de comunicação mantendo o indivíduo ativo e participante, promovendo a busca de melhores empregos e renda, facilitando a utilização dos serviços de saúde e a receptividade aos programas educacionais e sanitários ${ }^{11}$.

Em pesquisa intitulada: O envelhecimento nas diferentes regiões do Brasil: uma discussão a partir do censo demográfico 2010, os autores também destacam que, de um modo geral, o nível educacional dos idosos brasileiros é notoriamente baixo, isso porque, os idosos tiveram poucas oportunidades de frequentar a escola na etapa da vida considerada oportuna e esperada ${ }^{14}$.

A taxa de analfabetismo corresponde ao percentual de pessoas com 15 anos ou mais de idade que não sabem ler e escrever pelo menos um bilhete simples no idioma que conhecem, na população total residente da mesma faixa etária, em 
determinado espaço geográfico. Segundo último censo do IBGE a taxa de analfabetismo entre idosos do município de Salgueiro-PE, de 60 a 69 anos corresponde a $37,5 \%$, entre 70 a 79 anos a $46 \%$ e mais de 80 anos a $57,1 \%$ sendo esta última idosos com maior taxa de analfabetismo ${ }^{15}$. Galisteu et al. ${ }^{16}$, destacam ainda que essa baixa escolaridade decorre do fato de que antigamente a escola era vista como lugar de elite ou mesmo inexistia a possibilidade de trabalhar e estudar e assim muitas pessoas mediante a necessidade de trabalhar deixavam o estudo para segundo plano.

Os resultados apontados nesse estudo sobre os questionamentos utilizados também foram semelhantes ao encontrado em outras pesquisas de satisfação dos usuários na atenção básica. Em estudo sobre a satisfação dos usuários no SUS ${ }^{17}$, em relação ao tratamento recebido os usuários também se posicionaram de forma positiva classifificando-o como bom (67,3\%). Corroborando com o estudo em pesquisa realizada pelo Ministério da Saúde ${ }^{18}$, foi relatado que $62,96 \%$ dos respondentes avaliaram o atendimento da equipe de enfermagem como "bom" ou "muito bom", $25,93 \%$ consideraram regular e 10\% avaliaram como "ruim" ou "muito ruim", $71,9 \%$ dos respondentes avaliaram o atendimento do médico como "bom" e "muito bom", $17,29 \%$ como "regular" e $9,92 \%$ como "ruim" ou "muito ruim". No estudo de Lobato ${ }^{19}$ quanto ao atendimento na UBS, os entrevistados classificaram o atendimento como bom, pela facilidade de acesso, no entanto, percebeu-se a insatisfação com o atendimento, principalmente na marcação de consultas médicas.

Santiago et al. ${ }^{13}$, descreveram em sua pesquisa sobre a satisfação da qualidade no atendimento nas USF no município de Recife- PE que o direito e a confidencialidade das informações tiveram grande satisfação dos usuários. As questões relacionavamse às informações repassadas pelo médico/enfermeiro sobre o problema de saúde e o tratamento $(92,6 \%)$ e a confiança no médico/enfermeiro para contar seus problemas de saúde $(91,6 \%)$. O mesmo autor relata ainda que a alta satisfação com o trabalho dos profissionais é um importante resultado para a ESF que tem no trabalho dos profissionais e na sua relação com a população o elemento primordial de sua legitimação na organização do sistema de saúde.

Em pesquisa sobre a satisfação dos usuários ${ }^{7}$, quanto aos critérios de humanização no atendimento a saúde, $69,5 \%$ confia em todos os profissionais de saúde da rede pública pelos quais foram atendidos. Segundo o autor, o bom atendimento, baseado na escuta do usuário, e o bom desempenho profissional propiciam o vínculo do usuário com o serviço de saúde permitindo que os profissionais conheçam seus pacientes e as prioridades de cada um, facilitando-Ihes o acesso.

Outros aspectos analisados foram em relação ao acesso a unidade através da marcação de consultas, sendo que a maioria dos idosos relataram nunca apresentar dificuldade nessas marcações. Alguns aspectos que não foram estudados são apontados como situações problemas do acesso na pesquisa de Pereira et al.20, entre eles: a "não abertura da UBS nos finais de semana" (1,5\%), "indisponibilidade de telefone quando a UBS está fechada" (2,6\%), "não obter consulta com menos de 24 horas" (15,5\%) e "tempo de espera na recepção maior que 30 minutos" $(20,4 \%)$. O mesmo autor descreve ainda que o "tempo de espera" é adotado como parâmetro para medir a barreira de acessibilidade organizacional sendo que os usuários entrevistados em sua pesquisa manifestaram insatisfação (20,4\%), indicando que a espera excede o limite estabelecido de 30 minutos. 
Nery et al. ${ }^{21}$ destacaram em sua pesquisa que o tempo de espera para o atendimento é apontado como um fator negativo no aspecto de satisfação com os serviços. Ainda no estudo realizado por Pereira et al. ${ }^{20}$, foram relatados que os usuários declaram tempo suficiente para consulta (84\%); responde claramente as perguntas $(82,9 \%)$, sendo que ao estabelecer uma relação entre esses dois itens cabe refletir sobre o espaço que é destinado à escuta das necessidades destes usuários, apontando que esta escuta pode se dar no sentido de abordagens voltadas para os aspectos biológicos, fato que pode ter contribuído para o usuário considerar o tempo da consulta suficiente.

Corroborando ainda com o estudo em pesquisa realizada pelo Ministério da Saúde ${ }^{18}$, dentre os entrevistados que receberam visita do ACS, $72,73 \%$ consideraram sua atuação "boa" ou "muito boa", 20,35\% consideraram sua atuação "regular" e 5,97\% "ruim" ou "muito ruim", mostrando então a satisfação dos usuários quanto a visita domiciliar tal como foi apontado nos resultados dessa pesquisa, podendo ser atribuída à proximidade do ACS com a comunidade.

No que se refere à prioridade no atendimento verifica-se que nem todos os usuários relataram possuí-la, corroborando com o estudo de Araújo; Valença; Rocha ${ }^{22}$, em sua pesquisa descrevendo que $41,3 \%$ dos entrevistados relataram não ter prioridade enfrentando a fila comum, como qualquer outro paciente em busca de assistência. Ressalta-se que o atendimento prioritário à pessoa idosa é um direito legalmente constituído devendo pois ser cumprido.

Percebeu-se ainda com a realização deste estudo que os usuários não possuem conhecimento sobre práticas educativas e não participaram de algum grupo na atenção básica, sendo importante destacar que essas práticas devem ser estimuladas correspondendo ao papel da atenção primária na prevenção, promoção, tratamento e manutenção da saúde. Freitas et al. ${ }^{23}$, afirmam ainda que o trabalho em grupo viabiliza a troca de experiências individuais, ato de extrema importância no combate à marginalidade social e na contribuição para autorrealização. Possibilita ainda, a construção de histórias coletivas, a partir do confronto de valores e ideologias, propiciando o enfrentamento de novos desafios.

Marin $^{24}$ em sua pesquisa discorreu que, para alguns entrevistados, a atenção à saúde ainda está voltada para os aspectos curativos, centrada na consulta médica e no atendimento à demanda, mesmo frente à proposta da ESF em que atuam, a qual visa a uma abordagem ampliada das condições de vida e saúde das pessoas, família e comunidade.

Paluskin; Vianna ${ }^{12}$ descreveram que alguns idosos relataram dificuldade de acesso, demonstrando que os serviços de atenção básica não têm funcionado como porta de entrada para todos os idosos que deles dependem, podendo estar vinculado à ideia de necessidade de procura do serviço apenas por motivo de doença. Paluskin; Valler; Viana ${ }^{25}$ relatam ainda que mudanças na estrutura do sistema de saúde estejam sendo perseguidas desde a implantação do SUS, onde observou-se que as ações de saúde buscadas pelos idosos e as próprias demandas e expectativas destes parecem centrar-se no atendimento individual, prestado pelo profissional médico e com enfoque curativo. 
No estudo de Dias; Tavares $^{26}$, dos idosos entrevistados, 11,7\% participavam de atividades educativas grupais, sendo a frequência representada por uma atividade $(79,7 \%)$, duas $(16,3 \%)$ e três $(3,6 \%)$. As atividades educativas grupais predominantes foram relacionadas a hipertensão arterial $(44,5 \%)$, ao idoso $(31,6 \%)$ e ao diabetes mellitus (18,4\%).

Araújo; Brito; Barbosa ${ }^{27}$ descrevem que as ações educativas são alternativas importantes para assegurar a autonomia do idoso e o envelhecer saudável, onde no modelo vigente ainda predominam ações curativas centradas no modelo biomédico havendo a necessidade de ampliar a promoção da saúde, na perspectiva da atenção básica, mediada pelo compromisso ético, social e político e envolver o idoso como partícipe de seu bem-estar, ampliando a participação social no SUS.

\section{CONCLUSÃO}

A partir do estudo foi possível observar que a maioria dos idosos está satisfeita quanto ao atendimento recebido pelas equipes da Estratégia de Saúde da Família, no entanto, deve-se repensar nas práticas voltadas para esse grupo populacional considerando não apenas as atividades assistenciais, mas também práticas preventivas e de promoção à saúde com uma abordagem multiprofissional e intersetorial que considere o idoso em sua totalidade direcionando atividades específicas para essa faixa etária.

A realização de pesquisas que abranjam um maior número de usuários também é necessária, buscando avaliar a satisfação em um grupo maior de indivíduos cadastrados nessas unidades, utilizando outros critérios, por exemplo, a aplicação do questionário no ambiente domiciliar visto que grande parte dos idosos possuem dificuldades de se locomover até as unidades e, portanto, não puderam participar do estudo.

Por fim, destaca-se que pesquisar sobre a satisfação dos usuários é fundamental para a gestão do serviço proporcionando a avaliação sobre a ótica dos usuários e indicação de decisões estratégicas em busca de melhoria na qualidade da atenção a saúde. Espera-se que gestores utilizem destes dados e desta prática de pesquisa, como método para conhecer o nível de satisfação dos seus usuários.

\section{REFERÊNCIAS}

1. Garuzi M, Achitti MCO, Sato CA, Rocha SA, Spagnuolo RS. Acolhimento na Estratégia Saúde da Família: revisão integrativa. Rev Panam Salud Publica. 2014;35(2):144-9.

2. Brasil. Ministério da Saúde. Secretaria de Atenção à Saúde. Departamento de Atenção Básica.Política Nacional de Atenção Básica. Brasília: Ministério da Saúde, 2012.

3. Brasil. Ministério da Saúde. Envelhecimento e saúde da pessoa idosa. Brasília: Ministério da Saúde, 2007. 
4. Brasil. Ministério da Saúde. Secretaria de Atenção à Saúde. Departamento de Atenção Básica. Envelhecimento e saúde da pessoa idosa. Brasília: Ministério da Saúde, 2006.

5. Albuquerque $A B$, Deveza $M$. Adesão ao tratamento na prática do Médico de Família e Comunidade e na Atenção Primária à Saúde. Porto Alegre: PromefArtmed. 2009; 3(4):41-72.

6. Brandão ALRBS, Giovanella L, Campos CEA. Avaliação da atenção básica pela perspectiva dos usuários: adaptação do instrumento EUROPEP para grandes centros urbanos brasileiros. Ciência \& Saúde Coletiva. 2013; 18(1):103-114.

7. Moimaz SAS, Marques JAM, Saliba O, Garbin CAS, Zina LG, Saliba NA. Satisfação e percepção do usuário do SUS sobre o serviço público de saúde. Physis Revista de Saúde Coletiva. 2010; 20 (4): 1419-1440.

8. Instituto Brasileiro de Geografia e Estatística. Estimativas da população residente no Brasil e unidades da federação com data de referência em 1ํ de julho de 2014. Disponível em: << http://www.ibge.gov.br/home/estatistica/populacao/estimativa2014/estimativa_dou.sh tm>>. Acesso em agosto de 2014.

9. Brasil. Ministério da saúde. Cadastro Nacional de Estabelecimentos de Saúde(CNES)/Departamento de Informática do Sistema Único de Saúde (DATASUS). Disponível em: < http://cnes.datasus.gov.br/Mod_Ind_Equipes.asp > . Acesso em setembro de 2014.

10. Pilger $\mathrm{C}$, Menon MH, Mathias TAF. Características sociodemográficas e de saúde de idosos: contribuições para os serviços de saúde. Rev. Latino-Am. Enfermagem set.-out. 2011.

11. Mastroeni MF, Erzinger GM, Mastroeni SSBS, Silva NN, Marucci, MFN. Perfil demográfico de idosos da cidade de Joinville, Santa Catarina: Estudo de base domiciliar. Rev Bras Epidemiologia. 2007; 10(2): 190-201.

12. Paskulin LMG, Vianna LAC. Perfil sociodemográfico e condições de saúde autoreferidas de idosos de Porto Alegre. Rev Saude Publica 2007; 41(5):757-768.

13. Santiago RF, Mendes ACG, Miranda GMD, Duarte, PO, Furtado, BMASM, Souza, WV. Qualidade do atendimento nas Unidades de Saúde da Família no município de Recife: a percepção do usuários.Ciência \& Saúde Coletiva. 2013;18(1):35-44.

14. Mafra SCT, Silva EP, Fonseca ES, Almeida AV, Freitas NC. O envelhecimento nas diferentes regiões do Brasil: uma discussão a partir do censo demográfico 2010. VI Workshop de análise ergonômica no trabalho. Envelhecimento, como pensar o trabalho, a sociedade e as cidades?.Disponível em:<<http://www.ded.ufv.br/workshop/docs/anais/2013/ Simone\% 20 Caldas\%20Tavares\%20Mafra\%20\%20\%20\%20\%20\%20\%20Tem\%C3\%A1tica\%20 Envelhecimento.pdf. 2013. 
15. Brasil. Ministério da saúde. Departamento de Informática do Sistema Único de Saúde (DATASUS). Disponível em:

<http://www2.datasus.gov.br/DATASUS/index.php?area=0206 >. Acesso em novembro de 2014.

16. Galisteu KJ, Facundim SD, Ribeiro RCHM, Soler ZASG. Qualidade de Vida de idosos de um grupo de convivência com amensuração da escala de Flanagan. Arq Ciênc Saúde. 2006 out/dez;13(4):209-214.

17. Castro, HCO, Machado LZ, Walter MIMT, Ranincheski SM, Schmidt BV, Marinho DNC et al. A Satisfação dos Usuários com o Sistema Único de Saúde (SUS). Sociedade em Debate.2008; 14(2): 113-134.

18. Ministério da saúde. Secretaria de Gestão estratégia e participativa. Relatório da Pesquisa de Satisfação dos Usuários do SUS quanto aos aspectos de acesso e qualidade percebida na atenção à saúde, mediante inquérito amostral. 2011.

19. Lobato MSA. Saúde do Idoso: reflexões sobre o atendimento e o nível de satisfação dos idosos inseridos no Programa HIPERDIA IV Jornada internacional de políticas públicas. Universidade Federal do Maranhão. São Luiz: Maranhão, 2013.

20. Pereira MJB, Curvo PA, Fortuna CM, Coutinho SS, Queluz MC, Campos LVO et al. Avaliação das características organizacionais e de desempenho de uma unidade de Atenção Básica à Saúde. Revista Gaúcha Enfermagem. 2011;32(1):48-55.

21. Nery MG, Marcelo VC, Dantas MAA. A Satisfação de Idosos Quanto à Estratégia Saúde da Família, a Partir da Atenção em Saúde Bucal. R bras ci Saúde.2010; 14(1):43-50.

22. Araujo VR, Valença AMG, Rocha AV. Saúde do Idoso na Atenção Básica de Saúde no Município de João Pessoa: o Olhar do Usuário. Revista brasileira ciências da Saúde. 2012; 16(2):195-204.

23. Freitas EV, PY I, Cançado FAX, Doll J, Gorzobi ML. Tratado de geriatria e gerontologia. 2. ed., Rio de Janeiro: Guanabara Koogan, 2006. 1573p.

24. Marin MJS, Marques APMF, Feres BOM, Saraiva AKH, Druzian S. A atenção à saúde do idoso: ações e perspectivas dos profissionais. Rev. Bras. Geriatr. Gerontol. 2008; 11(2).

25. Paskulin LMG, Valer, DB, Vianna LAC. Utilização e acesso de idosos a serviços de atenção básica em Porto Alegre (RS, Brasil). Ciência \& Saúde Coletiva.2011; 16(6):2935-2944.

26. Dias FA, Tavares DMS. Fatores associados à participação de idosos em atividades educativas grupais. Rev Gaúcha Enferm. 2013;34(2):70-77.

27. Araujo MAS, Brito $C$, Barbosa MA. Atenção básica à saúde do idoso no Brasil: limitações e desafios. Geriatria \& Gerontologia. 2008;3(2):122-125. 
Recebido: 08 agosto 2017. Publicado: 22 agosto 2017

Correspondência: Alaine Santos Parente. Rua Poeta Levino Neto, 933, bairro Prado, Salgueiro-PE, CEP: 56000-000. Email: alainefisio@hotmail.com

Conflito de Interesses: os autores declararam não haver conflito de interesses

(C) This is an Open Access article distributed under the terms of the Creative Commons Attribution License, which permits unrestricted use, distribution, and reproduction in any medium, provided the original work is properly cited. 